\title{
Analysis of in vivo cell migration in mosaic zebrafish embryos.
}

Arthur Boutillon ${ }^{1}$, Florence A. Giger ${ }^{2}$, Nicolas B. David ${ }^{1}$

1. Laboratory for optics and biosciences, Ecole polytechnique, CNRS, INSERM, Université Paris-Saclay, 91128 Palaiseau cedex, France.

2. Centre for Developmental Neurobiology and MRC CNDD, IoPPN, Guy's Campus, King's College London, London SE1 1UL, UK

\begin{abstract}
Being optically clear, the zebrafish embryo is a nice model system to analyse cell migration in vivo.

This chapter describes a combination of injection and cell transplant procedures that allows creation of mosaic embryos, containing a few cells labelled differently from their neighbours. Rapid 5D confocal imaging of these embryos permits to simultaneously track and quantify the movement of large cell groups, as well as analyse the cellular or subcellular dynamics of transplanted cells during their migration. In addition, expression of a candidate gene can be modified in transplanted cells. Comparing behaviour of these cells to control or neighbouring cells allows determination of the role of the candidate gene in cell migration. We describe the procedure, focusing on one specific cell population during gastrulation, but it can easily be adapted to other cell populations and migrating events during early embryogenesis.
\end{abstract}

\section{Introduction}

Cell migration is key to build, shape, repair and defend an organism. It has been extensively studied in vitro, providing invaluable knowledge on its cellular and molecular bases $[1,2]$. It, however, clearly appeared in the past few years that a number of cells behave quite differently in vitro and in vivo [3, 4]. This likely stems from the more complex environment they encounter (3D extracellular matrix of 
varying stiffness, neighbouring cells, guidance cues) leading for instance cells that would use only lamellipodia on a flat surface to use a wide array of cell processes (lamellipodia, pseudopodia of varying length and shapes, blebs...) for their in vivo displacements [5]. Many cells furthermore display collective behaviours, their migration depending on interactions with neighbouring cells $[6,7]$. Understanding in vivo cell migration thus implies direct analysis of cells moving in their physiological environment.

Doing so obviously requires optically clear systems permitting cell observation without further manipulation or dissection. Cells also need to be labelled, usually through the expression of fluorescent proteins. This can be achieved using transgenic lines expressing reporter constructs in specified cell types. However such a strategy often leads to label a whole cell population, when labelling sparse, isolated cells, is key to good imaging, in particular for analysing cell contours, or membrane protrusions which cannot be seen if neighbouring cells are similarly labelled $[8,9]$. Creating mosaic embryos, containing only one or few cells differing from surrounding cells, is thus crucial for precise analysis of cell dynamics. Mosaic embryos furthermore permit to test consequences of activation/repression of genes/pathways on migration of individual cells. Comparing the tracks and behaviour of these cells with surrounding control cells under the same experimental conditions allows determination of the role of a particular gene in cell migration [10]. We here describe a procedure, based on injections and cell transplants, to create mosaic zebrafish embryos during gastrulation and image migrating cells.

Gastrulation is the developmental stage during which cells first migrate, to organise the embryo in different germ layers and set up the basis of the future body plan [11]. This involves a wide array of cell migrations, including random walks, directed migrations and collective movements. The fish embryo, being optically clear, offers direct access to these processes [12]. Among the diverse movements taking place during gastrulation, cells of the organiser (termed 'shield' in zebrafish) internalise and migrate collectively toward the animal pole of the embryo, forming the prechordal 
plate $[9,13,14]$. We combine injections of RNAs encoding fluorescently tagged proteins, cell transplants and in vivo imaging, to analyse the cellular bases of this migration. We have successfully used the proposed protocol to describe behaviour of wild-type cells, dissect the pathways controlling their motility and orientation, as well as assess the importance of candidate genes in regulating cell migration in vivo [14-16]. The proposed protocol can be used to test the potential implication of any candidate gene in controlling cell migration in vivo. It can furthermore be easily adapted to analyse other cell types, or other developmental stages.

\section{2- Material}

\subsection{RNA injection}

1. Stereo-microscope.

2. Glass capillaries (Internal Diameter $=0.58 \mathrm{~mm}$, External Diameter $=1 \mathrm{~mm}$ ).

3. Needle puller (KOPF Vertical pipette puller, model 720, David Kopf Instruments, Tujunga, California, USA).

4. Injection mould (Figure 1a, 3D model available as a .stl file on request; commercially available from adaptive science tools, model TU1).

5. Injector (Eppendorf, Transjector 5246).

6. Manipulator (Narishige, model M-152).

7. $20 \mu \mathrm{l}$ microloader tips (Eppendorf), to load injection needles.

8. Plastic Pasteur pipettes for transferring embryos.

9. Embryo Medium (EM, Zebrafish book [17]).

10. RNAs: mRNAs are prepared in vitro, using the mMessage mMachine kit (Ambion) to produce fully capped RNAs.

11. Sterile-filtered water (Sigma, W3500) for diluting RNAs. 
12. Injection needle: needles should be long enough to reach the embryo within its chorion, without creating too large a hole in the chorion. It however should be short enough to keep some rigidity, which is key to piercing the chorion and the embryo. Figure $1 \mathrm{~b}$ shows a typical injection needle. To prepare a needle, mount a glass capillary (Internal Diameter $0.58 \mathrm{~mm}$ ) on the needle puller. Adjust temperature and pulling strength (see Note 1). Test different settings until obtaining the desired needle shape (check needles under a dissection scope). Once the puller is correctly set, prepare several dozen injection needles. Carefully store them in a Petri dish, secured on a putty band and seal the dish with parafilm to avoid dust.

13. Injection plate: melt $0.5 \mathrm{~g}$ of agarose in $50 \mathrm{ml} \mathrm{EM}$ in a microwave to prepare a $1 \%$ agarose gel. Pour it in a $90 \mathrm{~mm}$ Petri dish. When the agarose is at $60^{\circ} \mathrm{C}$, gently place the injection mould so that it floats. Let the agarose solidify. Remove the mould with forceps, add some EM to prevent the gel from drying and store at $4^{\circ} \mathrm{C}$ up to a month.

\subsection{Shield to shield transplant}

1. Fluorescent stereo-microscope.

2. Glass capillaries (Internal Diameter $=0.78 \mathrm{~mm}$, External Diameter $=1 \mathrm{~mm}$ ).

3. Microforge (Narishige, model MF900).

4. Microgrinder (Narishige, model EG-44).

5. Transplant mould (Figure 1d, 3D model available as a .stl file on request; commercially available from adaptive science tools, model PT1).

6. Eyelash mounted on a stick.

7. Micromanipulator (Leica, mechanical micromanipulator).

8. Microinjector (Narishige, model IM-9B).

9. Fine tweezers for dechorionation (Dumont Fine Science Tools).

10. $35 \mathrm{~mm}$ Petri dishes. 
11. Fire-polished glass Pasteur pipettes, to manipulate dechorionated embryos: approach the tip of the pipette close to the flame of a lighter until the glass slightly melts. Be careful not to melt it too much, otherwise the pipette opening may end up too small and embryos will be damaged when drawn into the pipette.

12. Penicillin / Streptomycin (Thermo Fisher Scientific): for transplanted embryos, use at $100 \mathrm{unit} / \mathrm{ml}$ Penicillin and $100 \mu \mathrm{g} / \mathrm{ml}$ streptomycin in EM.

13. Transplant needle: mount a glass capillary (Internal Diameter $0.78 \mathrm{~mm}$ ) on the needle puller and pull the capillary. The stretched part of the needle should be around $1.5 \mathrm{~cm}$ (Figure 1e). Test different settings until obtaining the desired shape and pull several dozen needles (see Note 1). Mount a needle on the microforge. Position the needle very close to the microforge's filament, at the point where its inside diameter is $25 \mu \mathrm{m}$. Briefly heat the filament: upon dilatation the filament touches the needle, the glass locally melts then breaks upon retraction of the filament. Using the microgrinder, grind the tip of the needle for one minute at maximum speed to form a $35^{\circ}$ bevel (Fig. 1e; see Note 2). To remove glass residues, mount the needle on a needle holder connected to a syringe (Fig. 1c) and wash the tip by briefly aspiring $2 \%$ hydrofluoric acid (toxic and highly corrosive, manipulate under a chemical fume hood) over $5 \mathrm{~mm}$. Repeat three times. Immediately rinse three times by aspiring acetone over $1 \mathrm{~cm}$. Store the needles on a putty band in a parafilm-sealed Petri dish.

14. Transplant plate: melt $0.5 \mathrm{~g}$ of agarose in $50 \mathrm{ml} \mathrm{EM}$ in a microwave to prepare a $1 \%$ agarose gel. Pour it in a $90 \mathrm{~mm}$ Petri dish. When the agarose is at $60^{\circ} \mathrm{C}$, gently place the injection mould so that it floats. Let the agarose solidify and remove the mould with forceps.

15. Transplant setup: around the fluorescent stereo-microscope, install the microinjector, connected to a needle holder mounted on the micromanipulator (Fig. 1f). 


\subsection{Mounting and imaging}

1. MatTek chamber (35 mm Dish, No. 1.5 Coverslip, 7 mm Glass Diameter, MatTek Corporation, Ashland, MA).

2. Microscopy: we use a Ti PFS (Nikon) inverted microscope equipped with a CSUX1-A1 (Yokogawa) spinning disc module, a 40x water immersion objective (plan Apo, N.A. = 1.15, Nikon), a Cobolt Calypso laser (491 nm, $100 \mathrm{~mW}$ ) and a Cobolt Jive laser (561 nm, $100 \mathrm{~mW}$ ) (Cobolt, 04-01 Series). The microscope is caged and heated using The Cube (Life Imaging Services) to maintain constant temperature and the sample is placed in a The Brick (life imaging Services) chamber to maintain constant hygrometry. Metamorph is used for acquisitions.

\subsection{Softwares for imaging and picture analysing}

1. Metamorph (Molecular Devices, LLC).

2. ImageJ.

3. XuvTool [18].

4. IMARIS (Bitplane).

5. Matlab (Mathworks).

6. R (R Foundation for Statistical Computing).

\section{3-Methods}

\subsection{Embryo injection}

1- $\quad$ Warm the injection plate by placing it at $28^{\circ} \mathrm{C}$ at least 30 minutes before injection.

2- $\quad$ Once fishes have laid, collect the embryos. Rinse them well to remove all the droppings and scales from the adults and harvest in EM in a $90 \mathrm{~mm}$ Petri dish. Transfer 50 embryos in an empty injection plate and squeeze them gently inside the grooves using forceps, without piercing the chorion. Orient the embryos with the animal pole up. Fill the plate with EM. 
3- Wear gloves while manipulating RNA to prevent contamination with RNAse, keep all solutions on ice. Prepare $4 \mu \mathrm{l}$ of $50 \mathrm{ng} / \mu \mathrm{l}$ Histone2B-mCherry RNA solution by diluting the stock RNA solution in sterile-filtered water. This solution will be injected in embryos used as hosts in the transplant. Prepare $4 \mu \mathrm{l}$ of $50 \mathrm{ng} / \mu \mathrm{l}$ Histone2B-mCherry RNA and $70 \mathrm{ng} / \mu \mathrm{l}$ LifeAct-GFP RNA. This will be injected in embryos used as cell donors in the transplant. To affect a particular pathway, it is possible to add other compounds to this solution, which will be present only in the transplanted cells. In particular, morpholinos or RNAs encoding dominant-negative or constitutively active forms of proteins can be used.

4- Using microloader tips, fill the injection needle with $2 \mu \mathrm{L}$ of RNA solution. Insert the needle in a needle holder, mounted on a manipulator and connected to the injector. Under the scope, delicately open the needle using forceps, either by scraping the tip or by breaking the very tip by pinching it. To test whether the needle is open, apply pressure with the injector and check that a drop of RNA solution forms at the tip. Then put the tip of the needle into the EM to prevent it from drying.

5- $\quad$ To prepare host embryos, inject half of the embryos at the 1-cell stage, with the H2BmCherry solution (Fig. 2a) (see Note 3). To do so, place an embryo close to the injection needle. Using the manipulator, pierce through the chorion and through the cell membrane so that the needle tip is inside the cell (Fig. 2b). Avoid piercing the yolk and injecting into the yolk. Inject $4 \mathrm{nl}$ (see Note 4) and remove the needle. The embryo should stay in the agarose groove. Repeat for 25 embryos and put the plate at $28^{\circ} \mathrm{C}$. To prepare donor embryos, let embryos reach the 4-cell stage (1 hpf), and inject $2 \mathrm{nl}$ of the H2B-mCherry and LifeAct-GFP solution into one of the four cells (Fig. 2c). Repeat for 25 embryos and put the plate at $28^{\circ} \mathrm{C}$. 


\subsection{Dechorionate}

1- Coat a $35 \mathrm{~mm}$ plate with $1 \mathrm{ml}$ of $1 \%$ agarose in EM. When the agarose has solidified, fill the plate with warmed EM $\left(28^{\circ} \mathrm{C}\right)$. Transfer embryos to be dechorionated with a plastic Pasteur pipette. 2- With two fine tweezers, carefully remove the chorion. This is done by pinching the chorion with one tweezer and delicately pulling on the scratch with the second tweezer (see Note 5). Repeat until the embryo is freed from the chorion. Tear the chorion gently and progressively to avoid crushing the embryo. Dechorionated embryos are very fragile and will not survive contact with air or plastic: for further steps, use fire-polished glass pipettes and agarose-coated plates.

3- $\quad$ Clean the plate by removing torn chorions and damaged embryos with a glass pipette then carefully place the plate at $28^{\circ} \mathrm{C}$.

\subsection{Shield to shield transplants}

1- $\quad$ Fill a transplantation plate with Penicillin / Streptomycin EM and warm at $28^{\circ} \mathrm{C}$.

2- Wash the eyelash and transplant needle with $70 \%$ ethanol. For the transplant needle, mount it on a needle holder connected to a syringe and draw ethanol on $2 \mathrm{~cm}$ (Fig. 1c). Empty the needle and dry it by drawing air. Do not dry by blowing, as this may result in dust getting stuck in the needle. 3- When embryos have reached the shield stage (6 hpf), select donor and host embryos under a fluorescent stereo microscope. Pick embryos displaying bright and homogeneous fluorescence in the shield. Use the eyelash to manipulate and sort embryos. With a fire-polished glass pipette transfer selected embryos into the wells of the transplantation plate, aligning vertically and side by side hosts and donors. Rotate the embryos with the eyelash in order to place the shield up, slightly tilted toward the needle (Fig. 3a,b).

3- Install the transplant needle on the needle holder of the transplant setup (see Note 6). Under the scope, orient correctly the bevel of the needle: if cells must be transplanted deep, turn the bevel downward; orient it upward to transplant cells superficially. Lower the needle into the EM and aspire 
EM over half of the stretched part of the needle (see Note 7).

4- $\quad$ Place a donor embryo in front of the needle and delicately insert the needle into the shield.

Be careful not to pierce nor be too close to the yolk. Gently draw up a few cells inside the needle (see Note 8). Using fluorescence, check that cells in the needle are labelled.

5- Place the corresponding host embryo in front of the needle and delicately insert the needle into the shield. Be careful not to approach the yolk too much. Gently blow the cells into the host, taking care not to add too much liquid with them. However, it may be necessary to keep expelling EM while moving the needle out of the embryo, in order for the cells not to stick to the needle. Be careful not to blow any air as this will damage the embryo. Use fluorescence to check that cells are now in the shield of the host embryo (Fig. 3c).

6- $\quad$ Repeat steps 4 and 5 until all host embryos have been transplanted. Remove donor and damaged embryos. Carefully place the plate in a $28^{\circ} \mathrm{C}$ incubator.

7- $\quad$ Clean transplant needle with water, as described in step 2.

\subsection{Mounting embryos}

Embryos are mounted in a small volume of warm agarose, which cools and thus solidifies rapidly. Being fast is thus crucial: prepare workbench before mounting, so that all required equipment is at hand. Do not mount more than 3 embryos at a time.

1- $\quad$ Prepare $1 \mathrm{ml}$ of $0.2 \%$ agarose in Penicillin / Streptomycin EM solution in a small glass vial and place it in a preheated $42^{\circ} \mathrm{C}$ hot block.

2- $\quad$ Select one to three embryos and draw them in a fire-polished pipette. While the embryos are in the pipette, put the MatTek plate under the scope and focus on the bottom of the well.

3- $\quad$ Drop the embryos in the $0.2 \%$ agarose solution without adding too much EM. Discard the remaining EM and draw the embryos back into the pipette. Take care to draw enough agarose after the embryos in order to fill the well before the embryos fall out. 
4- Blow a drop of agarose and the embryos into the MatTek well. Take care not to let the embryos touch air or the border of the well. Make sure the well is completely filled with agarose (see Note 9).

5- $\quad$ Depending on room temperature, the agarose will set in about 1 minute, during which embryos should be oriented using the eyelash. While orienting the embryo, be careful to touch it only on the blastoderm and not on the yolk, which is very fragile. Depending on the type of microscope used for imaging (upright or inverted), place the shield upward or downward, against the glass bottom. In the latter case, use fluorescence to spot transplanted cells and orient the embryo properly.

6- Wait a few minutes for the agarose to set completely, then add a drop of Penicillin / Streptomycin EM to prevent it from drying.

\subsection{Imaging}

1- $\quad$ Preheat the microscope cage at $28^{\circ} \mathrm{C}$ at least 30 minutes before starting imaging.

2- $\quad$ Put the MatTek plate under the spinning-disc microscope and fill it with Penicillin /

\section{Streptomycin EM.}

3- $\quad$ Set the hygrometry module to $80 \%$ relative humidity. In absence of hygrometry control, place a water-soaked tissue to keep constant moisture. This will prevent the EM from evaporating and the agarose from drying.

4- Using a 40x long range objective, spot an embryo and focus on labelled cells. Tune the laser power and exposure time to optimise image dynamics for both channels. Specified RNA doses should lead to bright GFP and mCherry signals, so that brief exposure times can be used ( $<100 \mathrm{~ms}$; see Note 10). This limits photobleaching and toxicity, and allows for high frame rates, compatible with acquiring large $\mathrm{z}$-stacks, at short time intervals ( 2 minutes). Using bright field, set the $\mathrm{X}$ and $\mathrm{Y}$ position, then the top and bottom of the z-stack, so that it encompasses the entire shield. Be careful 
to consider that the embryo is curved and that cells may therefore not migrate in a straight z plane. It may thus be useful to enlarge the z-stack in order to have the whole prechordal plate encompassed in the stack for the duration of the timelapse. To get a larger field of view, image several positions that will be stitched with the XuvTool software [18]. Neighbouring stacks require a 5-10\% overlap. 5- $\quad$ Repeat step 4 for every embryo in the imaging plate then set the time interval to 2 minutes and z-step to $2 \mu \mathrm{m}$. Launch the timelapse for two hours. Be careful that total acquisition time at each time step does not exceed 2 minutes.

\subsection{Data analysis}

A. Quantification of actin-rich protrusion orientation

1- $\quad$ Load images in ImageJ using the Bioformat plugin (Fig. 4a).

2- $\quad$ Using the movement of labelled cells, determine the general direction of migration then rotate the movie using the "Rotate" function of ImageJ to set the direction angle to $0^{\circ}$.

3- On the green channel, for each time step and each transplanted cell, look for actin-rich protrusions. We consider all protrusions exceeding $5 \mu \mathrm{m}$ in length. If present, measure their 2D orientation using the "Angle" tool. Draw a straight line from the centroid of the cell toward the stem of the protrusion and measure the angle relative to the general direction of migration (Fig. 4b).

4- $\quad$ Use $R$ and the circular package for data analysis and representation. If angles are measured over $0-180^{\circ}$, classic statistical tests can be used to compare two distributions, like the Kolmogorov-Smirnov test (ks.test). If angles are measured over $0-360^{\circ}$, circular statistical tests, like Watson's two sample test (watson.two.test), should be used. Data are best presented as a rose diagram (rose.diag) (Fig. 4c).

B. Cell tracking 

camera used for imaging (Fig. 4d).

2- Use the "Spot" function to locate and track nuclei. Set object size to $10 \mu \mathrm{m}$, which is the average nucleus size at this developmental stage. Due to lower axial resolution, nuclei may appear elongated in the axial dimension. It is therefore best to allow ellipsoidal shape, with 15 $\mu \mathrm{m}$ in the $\mathrm{Z}$ axis. Filter on spot quality to remove false positive. Filter on red intensity to discriminate between ectoderm cells and migrating prechordal plate cells, which, being deeper in the embryo, appear dimmer. Prechordal plate cells migrate at about $3+/-0.8 \mu \mathrm{m} / \mathrm{min}[14]$, hence allow $9 \mu \mathrm{m}$ as the maximal distance between consecutive time steps. Allowing gaps over one or two time points provides longer continuous tracks, without introducing too many track errors. Once automatic tracking is done, visually check tracks and manually correct them if necessary (Fig. 4e).

3- $\quad$ Export the results as a .csv file and process with MatLab. We use custom made Matlab routines to compute instant speed, persistence, coherence, orientation of movement, etc. for each cell. Routines are available on request (Fig. 4f,g).

\section{4- Notes}

1- Injections and transplants are technically demanding steps, which highly depend on needles quality. Pulling needles of the right shape (see Fig. 1b,e for reference) requires fine tuning of parameters but generally, to get short and sharp needles for injection, the temperature should be set low and the tension high. On the contrary, for long transplant needles, temperature should be high and tension low.

2- To ease penetration in embryos, which can be difficult at late developmental stages, it is possible to add a barb at the tip of transplant needles. Install a transplant needle on the microforge with the tip $50 \mu \mathrm{m}$ above the filament. Heat the filament, lower the needle to briefly touch the 
filament and immediately pull back. By melting the very tip of the bevel, this will stretch a glass barb. If the barb is too long, break it with forceps (Fig. 1e, insert). Pay attention that this procedure may reduce the diameter of the needle opening.

3- Embryos meant to be hosts are injected at the 1-cell stage. Injected RNAs will diffuse in the cytoplasm, leading most cells to be labelled, but with varying intensities, depending on how much RNA they inherited. On the contrary, in donor embryos it is important that all labelled cells have inherited similar levels of RNAs, so that all transplanted cells are similar. Hence, donors are injected at the 4-cell stage, since the RNA will diffuse in a smaller volume and be distributed homogeneously into the daughter cells.

4- Some calibrate injection volume by injecting in an oil drop and measuring the diameter of the created droplet. Although theoretically precise, this technique implies that injection conditions are constant from one embryo to another. Experience proves this to be false, resistance to fluid flow in the needle varying largely from one embryo to another, due to differences in the position of the needle in the embryo, and partial, transient, clogging of the needle. To get reproducible injections, it is thus preferable to visually control the volume of injected liquid, which can be seen as a clearer droplet in the cytoplasm. By comparing the size of the droplet to the rest of the embryo, it is possible to have a good estimation of the injected volume (Fig. 2a,c). Phenol red may be added to the injection solution to better visualise the droplet.

5- While dechorionating, be very careful when first pinching the chorion, since this is when the embryo has the most chance to be crushed. In particular, for injected embryos, the chorion is pierced at the injection spot and embryos have a tendency to squeeze through this hole if chorion is brutally pinched.

6- Oil may be used instead of air in the transplant system. Being inelastic, oil provides a more reactive setup. Entirely fill the microinjector, the tubing and the needle with oil. Use the oil setup as described for the air setup. One drawback is that, to be efficient, the whole system must be 
purged of any air bubble, which may be difficult. Furthermore, needles filled with oil tend to get dirty and thus cannot be reused as many times as when used with air.

7- Be careful to have the right amount of liquid in your needle: enough to draw and blow cells without contact with air, but not too much, otherwise the system will respond with a delay and sudden jolts. The interface between air and embryo medium must be kept in the stretched part of the needle.

8- Be careful not to draw up yolk in the needle nor to draw up too much liquid after the cells, since injecting yolk or large volumes of liquid appear toxic to the host. Yolk can be seen as a transparent, non-fluorescent mass in the needle.

9- Once embryos have been deposited in the well, and before orienting them, briefly rinse the glass transfer pipette to avoid agarose solidifying into it. Otherwise, although not visible, agarose will partially clog the pipette and likely harm the next embryo that will be mounted.

10- Laser light and emitted fluorescent light are partially absorbed and diffracted by the sample, leading to signal attenuation when imaging deep. We use a custom Metamorph journal to compensate for this loss of signal. Exposure time is linearly increased while getting deeper in the sample, so that exposure time is doubled over the entire stack.

\section{Acknowledgement}

This work was supported by grant PJA 20151203256 for Fondation ARC pour la Recherche sur le Cancer, and grant ANR-15-CE13-0016-02 from Agence Nationale de la Recherche.

\section{References}

1. Horwitz R, Webb D (2003) Cell migration. Curr Biol 13:R756-9. doi: http://dx.doi.org/10.1016/B978-0-12-394447-4.20070-9 
2. Vicente-Manzanares M, Horwitz AR (2011) Cell migration: an overview. Methods Mol Biol 769:1-24. doi: 10.1007/978-1-61779-207-6_1

3. Lämmermann T, Sixt M (2009) Mechanical modes of "amoeboid" cell migration. Curr Opin Cell Biol 21:636-44. doi: 10.1016/j.ceb.2009.05.003

4. te Boekhorst V, Preziosi L, Friedl P (2016) Plasticity of Cell Migration In Vivo and In Silico. Annu Rev Cell Dev Biol 3228361:1-28. doi: 10.1146/annurev-cellbio-111315-125201

5. Ridley AJ (2011) Life at the leading edge. Cell 145:1012-22. doi: 10.1016/j.cell.2011.06.010

6. Haeger A, Wolf K, Zegers MM, Friedl P (2015) Collective cell migration: Guidance principles and hierarchies. Trends Cell Biol 25:556-566. doi: 10.1016/j.tcb.2015.06.003

7. Theveneau E, David NB (2014) [Collective cell migrations]. Med Sci (Paris) 30:751-7. doi: $10.1051 /$ medsci/20143008012

8. Row RH, Maître J-LL, Martin BL, et al (2011) Completion of the epithelial to mesenchymal transition in zebrafish mesoderm requires Spadetail. Dev Biol 354:102-10. doi: 10.1016/j.ydbio.2011.03.025

9. Montero J-A, Carvalho L, Wilsch-Bräuninger M, et al (2005) Shield formation at the onset of zebrafish gastrulation. Development 132:1187-1198. doi: 10.1242/dev.01667

10. Liang C-C, Park AY, Guan J-L (2007) In vitro scratch assay: a convenient and inexpensive method for analysis of cell migration in vitro. Nat Protoc 2:329-333. doi: 10.1038/nprot.2007.30

11. Solnica-Krezel LL, Sepich DS (2012) Gastrulation: Making and Shaping Germ Layers. Annu Rev Cell Dev Biol 28:687-717. doi: 10.1146/annurev-cellbio-092910-154043

12. Solnica-Krezel L, Stemple DL, Driever W (1995) Transparent things: cell fates and cell movements during early embryogenesis of zebrafish. BioEssays 17:931-9. doi: 
10.1002/bies.950171106

13. Kai M, Heisenberg C-P, Tada M (2008) Sphingosine-1-phosphate receptors regulate individual cell behaviours underlying the directed migration of prechordal plate progenitor cells during zebrafish gastrulation. Development 135:3043-51. doi: 10.1242/dev.020396

14. Dumortier JG, Martin S, Meyer D, et al (2012) Collective mesendoderm migration relies on an intrinsic directionality signal transmitted through cell contacts. Proc Natl Acad Sci U S A 109:16945-50. doi: 10.1073/pnas.1205870109

15. Dumortier JG, David NB (2015) The TORC2 Component, Sin1, Controls Migration of Anterior Mesendoderm during Zebrafish Gastrulation. PLoS One 10:e0118474. doi: 10.1371/journal.pone.0118474

16. Dang I, Gorelik R, Sousa-Blin C, et al (2013) Inhibitory signalling to the Arp2/3 complex steers cell migration. Nature 503:281-4. doi: 10.1038/nature12611

17. Westerfield M (2000) The zebrafish book. A guide for the laboratory use of zebrafish (Danio rerio), 4th ed., U.

18. Emmenlauer M, Ronneberger O, Ponti A, et al (2009) XuvTools: free, fast and reliable stitching of large 3D datasets. J Microsc 233:42-60. doi: 10.1111/j.1365-2818.2008.03094.x

\section{Figure legends}

Figure 1: Injection and transplant equipment. (a) 3D model of the injection mould used to prepare injection agarose plates. Dimensions are in $\mathrm{mm}$. Model is available as a .stl file. (b) Typical injection needle. (c) Needle holder connected to a syringe, used to rinse transplant needles. (d) 3D model of the transplant mould used to prepare transplant agarose plates. Dimensions are in $\mathrm{mm}$. Model is 
available as a .stl file. (e) Typical transplant needle. (f) General view of the transplant setup.

Transplant needle is inserted in a needle holder, mounted on a mechanical micromanipulator. The needle is connected to a microinjector.

Figure 2: Injection procedure. (a) Schematic of the injection procedure at the 1-cell stage. The injected volume on the schematic represents $4 \mathrm{nl}$. (b) 1-cell stage embryo maintained in an agarose groove. Injection needle is in the cell. (c) Schematic of the injection procedure at the 4-cell stage. The injected volume on the schematic represents $2 \mathrm{nl}$.

Figure 3: Shield to shield transplant procedure. (a) Schematic of the transplant procedure. Embryos are maintained in individual wells, oriented with the shield up, slightly tilted toward the needle. The needle is used to draw cells from the shield of a donor embryo and inject them in the shield of a host embryo. (b) Bright field image of a host embryo in an agarose well. (c) This host embryo was injected at the 1-cell stage with RNAs encoding Histone2B-mCherry. All nuclei are thus labelled in red. A few cells expressing LifeAct-GFP were transplanted into the shield.

Figure 4: Data analysis. (a) Transplanted cells expressing LifeAct-GFP. Large actin-rich protrusions are easily visible. (b) Measure of protrusion orientation. Image has been rotated so that the $x$-axis is aligned with migration movement. (c) Rose plot of the angular distribution of protrusions. (d) The host embryo expresses Histone2B-mCherry, labelling all nuclei in red. Dotted line delineates the limit between prechordal plate cells and ectodermal cells. (e) Tracks of prechordal plate cells, obtained in IMARIS. Upper part, XY view; lower part, XZ view. (f-g) Raw data from cell tracks are imported and processed in Matlab, to quantify migration properties. Here, distribution of average instant speed (f) of all tracked cells and distribution of cell persistence (g), calculated over 5 time steps. 


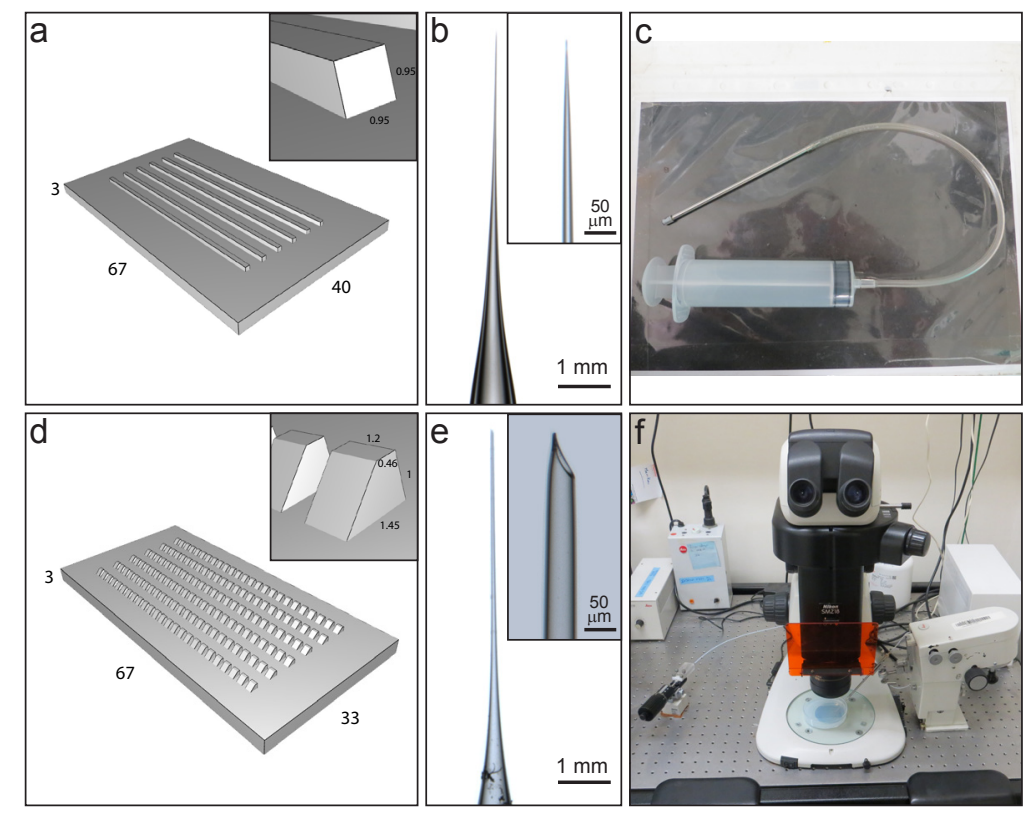




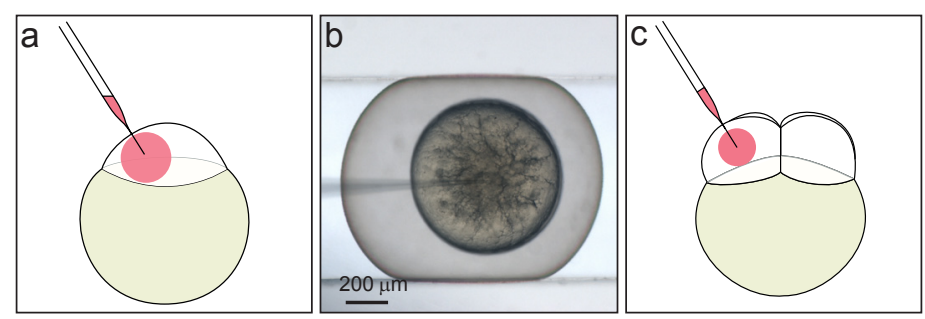




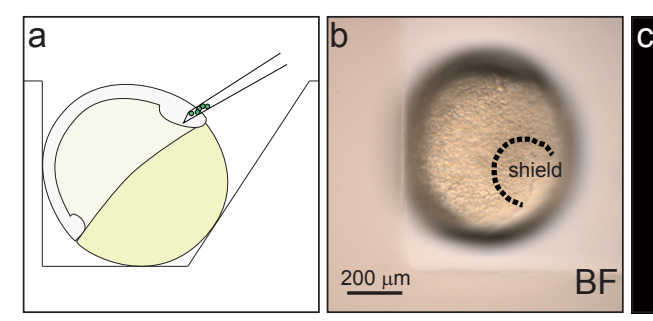



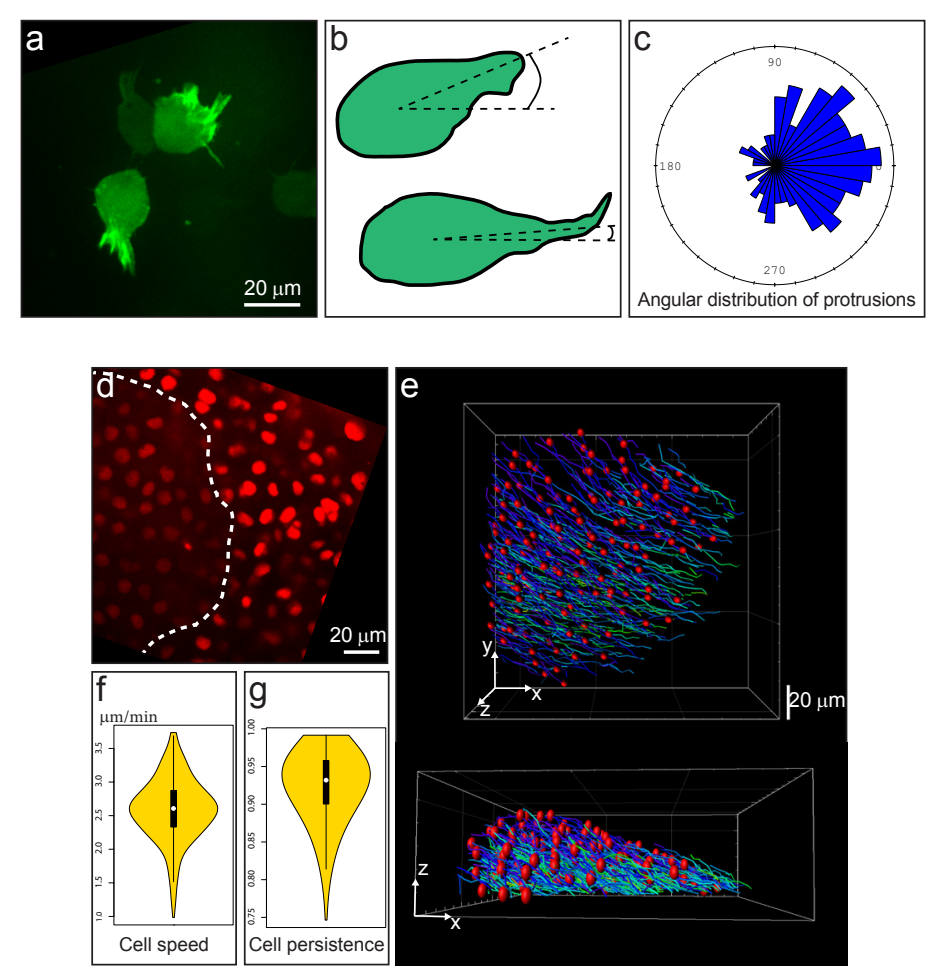